\title{
A case of biatrial thrombus in a patient with mitral stenosis presenting as systemic and pulmonary embolism
}

\author{
HARSHWARDHAN KHANDAIT ${ }^{1}$, Vinod Khandait ${ }^{1}$, and Madhuri Holay ${ }^{1}$ \\ ${ }^{1}$ Government Medical College and Hospital Nagpur
}

May 6, 2020

\begin{abstract}
Rheumatic heart disease is a sequelae of rheumatic fever. Mitral stenosis in rheumatic heart disease is common and leads to left atrial dilation and atrial fibrillation. Atrial fibrillation predisposes to thrombus formation in the left atrium and its appendage and is associated with embolic complications. Systemic embolism is frequent and associated with considerable mortality, especially in the developing world. Pulmonary embolism is rarely documented. Herein we report an extremely rare case of biatrial thrombus in a patient with rheumatic heart disease presenting as systemic as well as pulmonary embolism. Keywords: rheumatic, biatrial, thrombus, embolism
\end{abstract}

A case of biatrial thrombus in a patient with mitral stenosis presenting as systemic and pulmonary embolism Harshwardhan Khandait ${ }^{1}$

Vinod Khandait ${ }^{2}$

Madhuri Holay ${ }^{3}$

${ }^{1}$ MBBS, Department of Internal Medicine, Government Medical College, Nagpur, Maharashtra, India.

${ }^{2} \mathrm{MD}$, DNB, Department of Internal Medicine, Government Medical College, Nagpur, Maharashtra, India.

${ }^{3} \mathrm{MD}$, Department of Internal Medicine, Government Medical College, Nagpur, Maharashtra, India.

Correspondence:

Vinod Khandait, Department of Internal Medicine, Government Medical College, Nagpur, Maharashtra, India.

Email-drkhandait@rediffmail.com

Address - Department of Internal Medicine, Government Medical College, Hanuman nagar, Nagpur, Maharashtra, India, 440003.

Contact number- +919373107740

Running head- Systemic and pulmonary embolism in mitral stenosis

\section{Hosted file}

CASE REPORT.docx available at https://authorea.com/users/317532/articles/447590-a-caseof-biatrial-thrombus-in-a-patient-with-mitral-stenosis-presenting-as-systemic-andpulmonary-embolism 

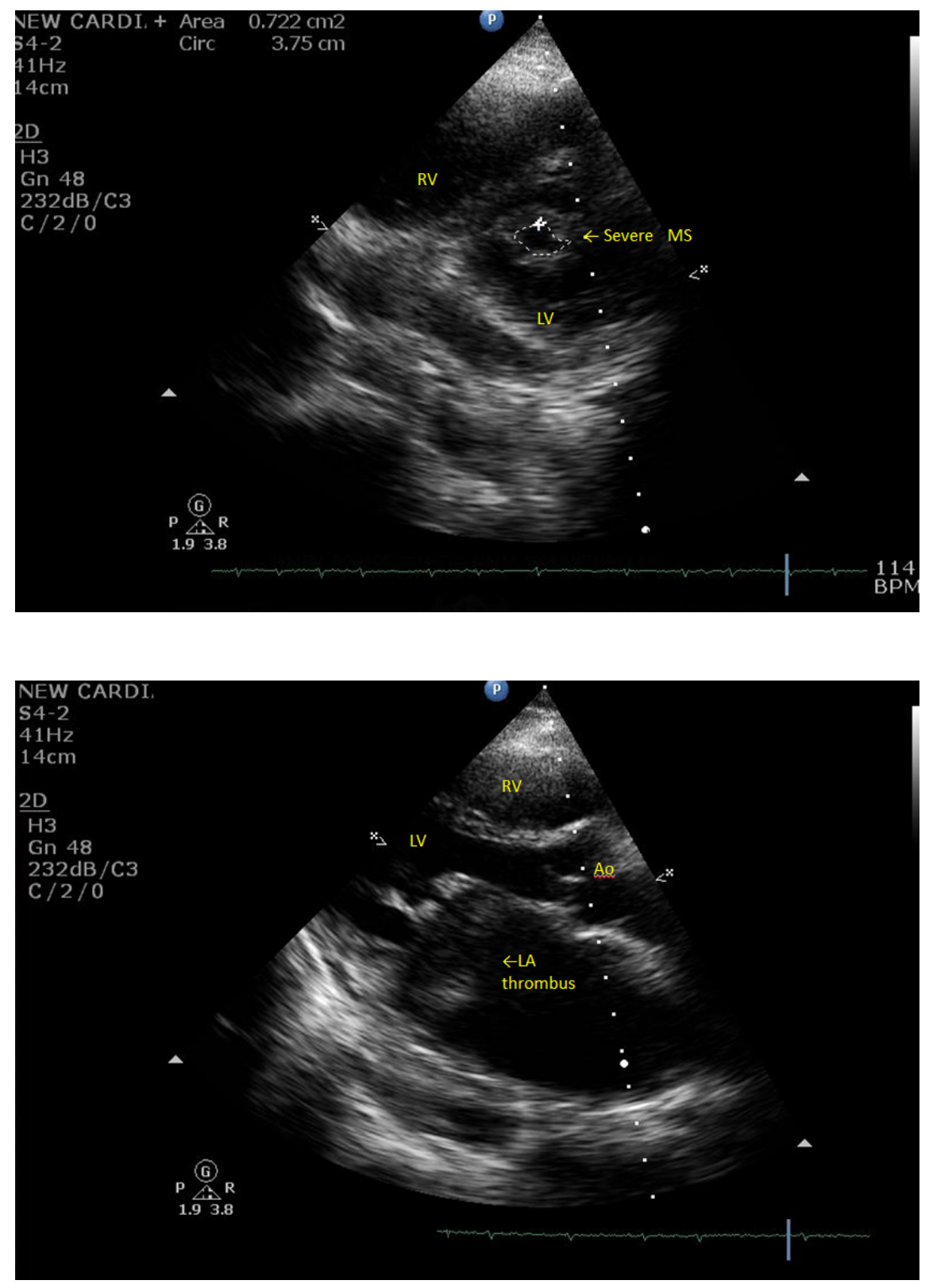

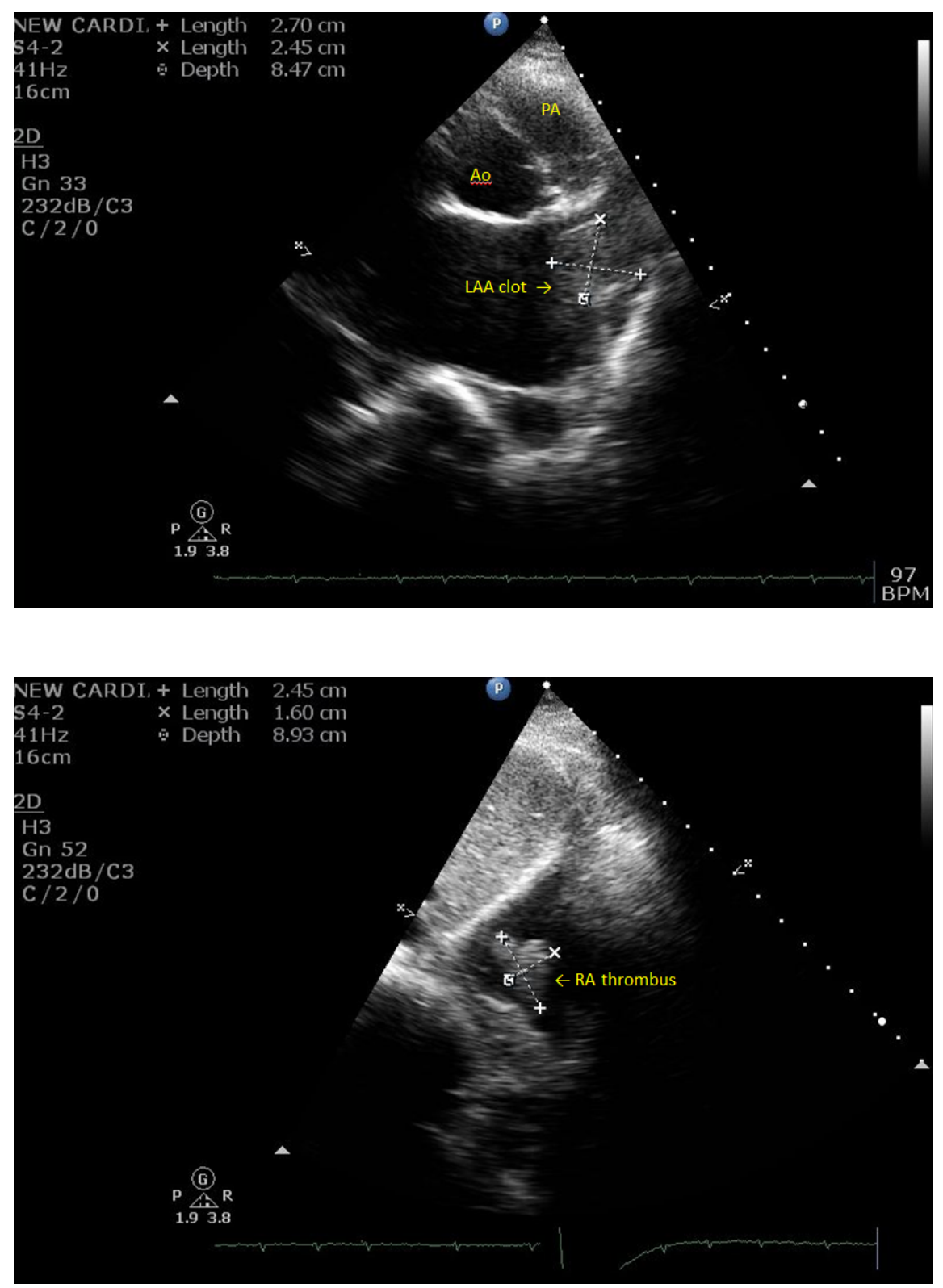

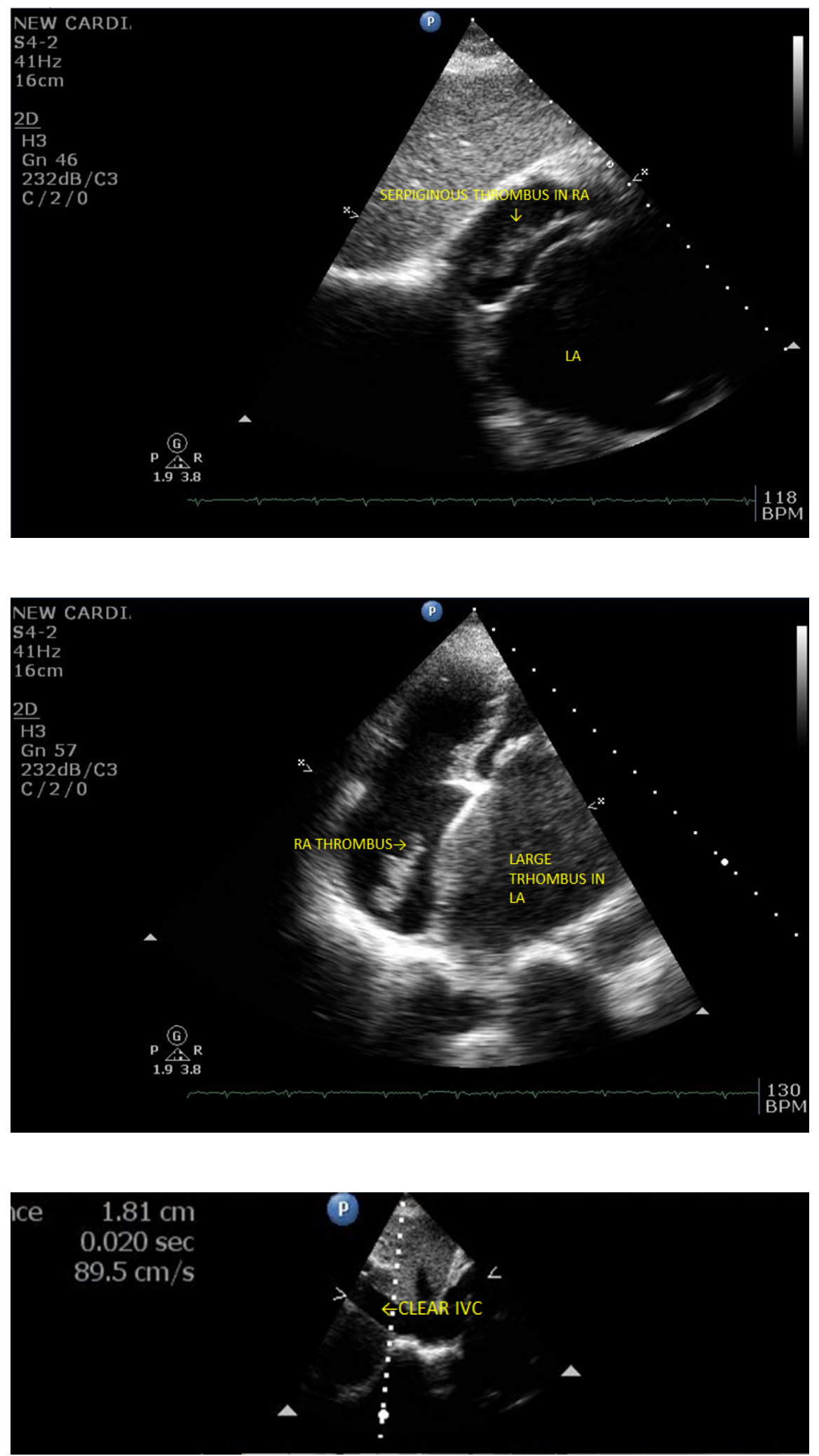

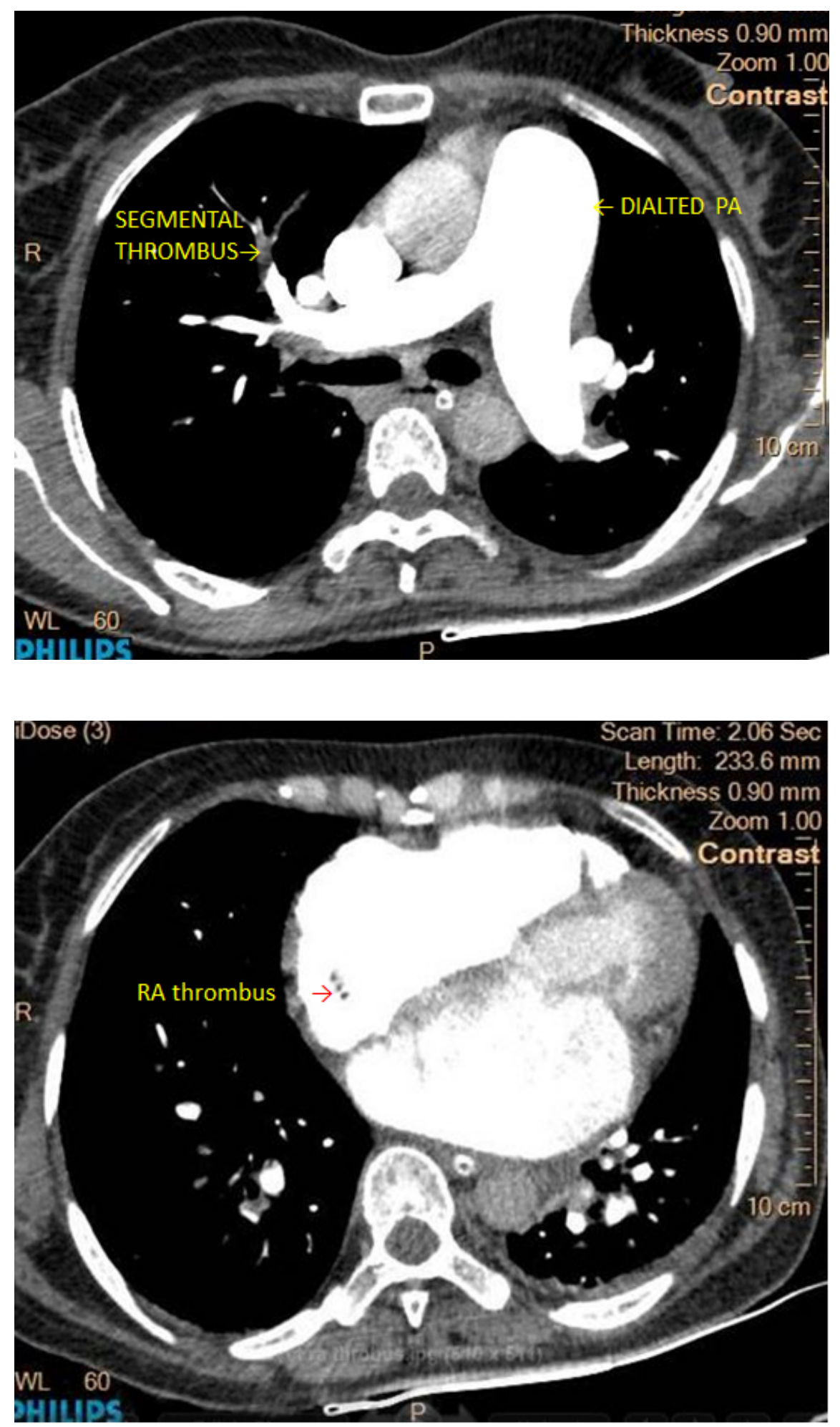


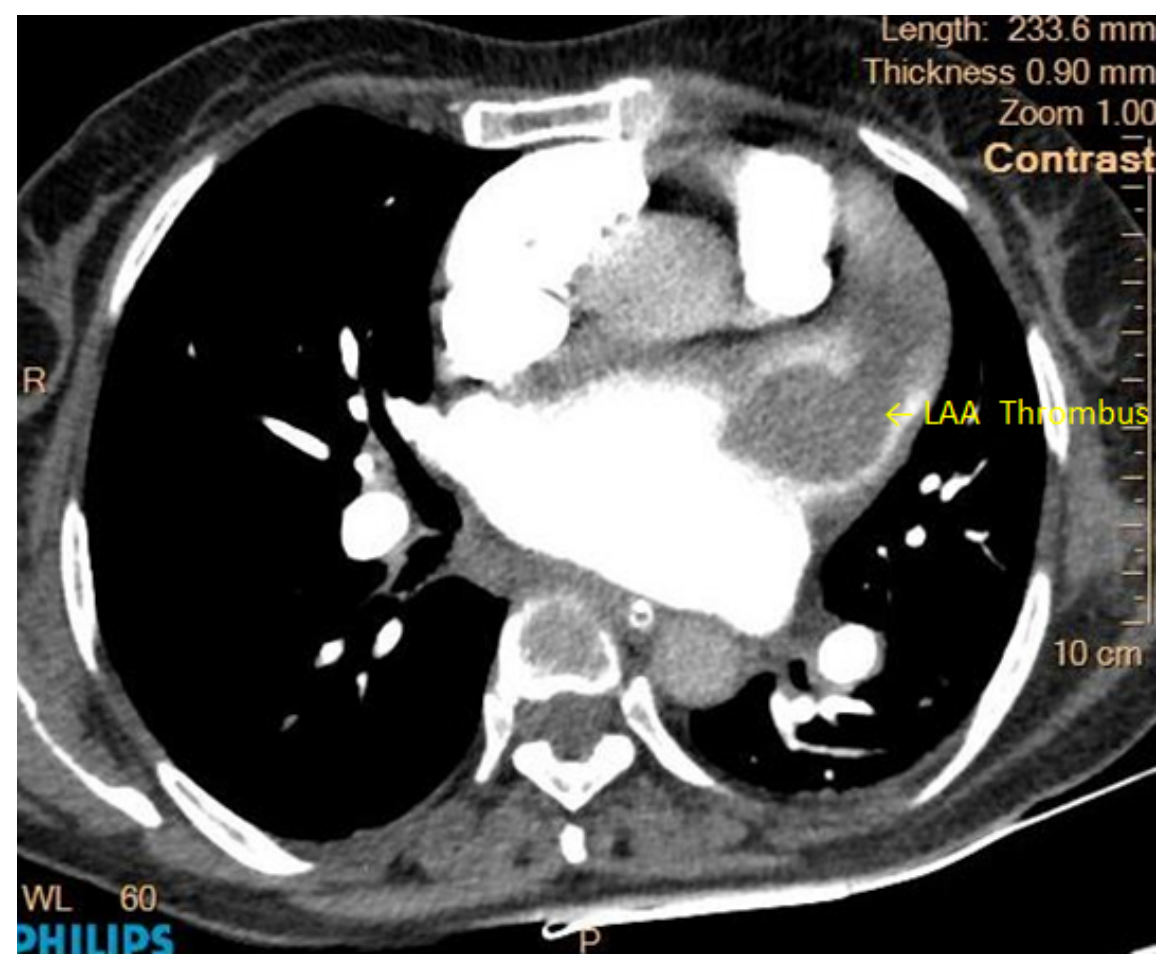

